\title{
Generation of human neutralizing monoclonal antibodies against the 2009 pandemic H1N1 virus from peripheral blood memory B lymphocytes
}

\author{
Hao Wang, Chi Ma, Yanlai Lu, Xu Ji, Yongsheng Pang, Fang Hua, Lianxian Cui, Denian Ba and Wei He
}

The $2009 \mathrm{H} 1 \mathrm{~N} 1$ influenza pandemic demonstrated the significance of a global health threat to human beings. Although pandemic $\mathrm{H} 1 \mathrm{~N} 1$ vaccines have been rapidly developed, passive serotherapy may offer superior immediate protection against infections in children, the elderly and immune-compromised patients during an influenza pandemic. Here, we applied a novel strategy based on Epstein-Barr virus (EBV)-immortalized peripheral blood memory B cells to screen high viral neutralizing monoclonal antibodies (MAbs) from individuals vaccinated with the 2009 pandemic H1N1 vaccine PANFLU.1. Through a massive screen of 13090 immortalized memory B-cell clones from three selected vaccinees, seven MAbs were identified with both high viral neutralizing capacities and hemagglutination inhibition (HAI) activities against the 2009 pandemic H1N1 viruses. These MAbs may have important clinical implications for passive serotherapy treatments of infected patients with severe respiratory syndrome, especially children, the elderly and immunodeficient individuals. Our successful strategy for generating high-affinity MAbs from EBV-immortalized peripheral blood memory B cells may also be applicable to other infectious or autoimmune diseases.

Cellular \& Molecular Immunology (2013) 10, 403-412; doi:10.1038/cmi.2013.25; published online 5 August 2013

Keywords: Epstein-Barr virus; hemagglutinin; memory B cells; neutralizing monoclonal antibody; 2009 pandemic influenza H1N1 virus

\section{INTRODUCTION}

The 2009 pandemic H1N1 influenza pandemic raised major public concern worldwide. It was reported that the outbreak of $2009 \mathrm{H} 1 \mathrm{~N} 1$ pandemic strain caused more than 60 million cases, more than 256000 of which required hospitalization. ${ }^{1-3}$ Although the $2009 \mathrm{H} 1 \mathrm{~N} 1$ pandemic influenza virus did not cause high mortality, an unusually high frequency of severe syndromes occurred in young children and the elderly. ${ }^{4}$ Fortunately, an efficient H1N1 vaccine, PANFLU.1, became available five months after the outbreak of 2009 influenza pandemic. One dose of non-adjuvant split-virion vaccine, containing $7.5 \mu \mathrm{g}$ hemagglutinin (HA), induced seroprotection in more than $90 \%$ of people between the ages 12 and 60 years. ${ }^{5}$ However, only $76.7 \%$ of children aged from 3 to 12 years and $80.3 \%$ in people over 60 years were seroprotected by this vaccine. ${ }^{5}$ Importantly, children and the elderly were particularly vulnerable to pandemic H1N1 influenza infection and suffered significantly higher mortality during the outbreak in $2009 .{ }^{1}$ Despite the rapid development of pandemic H1N1 vaccines and the fast-tracked approval processes, these products were not available for large-scale use until the end of the 2009 pandemic. Therefore, during an outbreak of pandemic influenza, it is critical to develop passive serotherapy to provide immediate protection against infections for young children, the elderly and medical personnel. Passive serotherapy can also be used as a cure for infected individuals with deadly respiratory syndrome, especially for immunocompromised patients.

During the outbreak of pandemic influenza A in 2009, insufficient amounts of human sera containing antibodies to pandemic influenza A H1N1 virus were available for the large population of infected patients. Therefore, we considered the generation of human monoclonal antibodies (MAbs) with high viral neutralizing capacities to influenza A H1N1 virus as an effective solution. A number of strategies have been reported to

Department of Immunology, Institute of Basic Medical Sciences, Chinese Academy of Medical Sciences and School of Basic Medicine, Peking Union Medical College, State Key Laboratory of Medical Molecular Biology, Beijing, China

Correspondence: Dr W He, Department of Immunology, Institute of Basic Medical Sciences, Chinese Academy of Medical Sciences and School of Basic Medicine, Peking Union Medical College, State Key Laboratory of Medical Molecular Biology, 5 Dong Dan San Tiao, Beijing 100005, China.

E-mail: heweiimu@public.bta.net.cn

Received: 28 March 2013; Revised: 13 May 2013; Accepted: 14 May 2013 
generate human MAbs, such as (i) hybridoma generation via fusing human B cells with a myeloma cell line; ${ }^{6,7}$ (ii) immortalizing B cells with Epstein-Barr virus (EBV); ${ }^{8,9}$ (iii) humanizing murine MAbs; $;^{10,11}$ (iv) selection of positive antibody fragments from phage-display libraries; ${ }^{12,13}(\mathrm{v})$ production of human MAbs from vaccinated transgenic mice carrying human immunoglobulin loci; ${ }^{14}$ and (vi) expression of human MAbs by protein engineering with the variable genes of MAbs from antibody-secreting plasma cells of infectious patients. ${ }^{4,15}$

The success of the $2009 \mathrm{H} 1 \mathrm{~N} 1$ vaccine PANFLU.1 in young adults provided a possibility for screening high viral neutralizing MAbs from vaccinated individuals. Accordingly, in the present study, we applied a novel strategy based on EBVimmortalized peripheral blood memory B cells to screen 2009 pandemic H1N1 influenza strain-specific neutralizing MAbs from vaccinated individuals who received PANFLU.1. Through a massive screen of 13090 immortalized B-cell clones from three selected vaccinees with hyperimmune sera, seven clones were identified to produce MAbs with both high viral neutralizing capacities and hemagglutination inhibition (HAI) activities. These EBV-immortalized memory B-cell clones can generate high viral neutralizing MAbs for clinical passive serotherapy of infected patients with severe respiratory syndrome, especially children, the elderly and immunodeficient individuals. This novel EBV-immortalized memory B-cell strategy may also be applicable to other infectious or autoimmune diseases.

\section{MATERIALS AND METHODS}

\section{Cell cultures}

The marmoset B-lymphoblastoid cell line B95-8 and 293T cells were cultured in RPMI 1640 medium containing $L$-glutamine (Invitrogen, Carlsbad, CA, USA) supplemented with $10 \%$ fetal calf serum (FCS) and penicillin-streptomycin at $37{ }^{\circ} \mathrm{C}$ in a humidified incubator with $5 \% \mathrm{CO}_{2}$. MDCK cells were cultured in DMEM medium (Invitrogen) supplemented with 10\% FCS. Spodoptera frugiperda (Sf9) cells were maintained in Sf-900 II SFM (Invitrogen).

\section{Volunteers enrolled in the experiments}

Fourteen young healthy young adults were vaccinated with 2009 H1N1 pandemic vaccine PANFLU.1 (Sinivac Biotech China) at the Institute of Basic Medical Science, Peking Union Medical College. Five healthy unvaccinated volunteers were used as negative controls.

\section{Isolation of memory $B$ cells}

Peripheral blood memory B cells were isolated using a magnetic isolation kit (Miltenyi, Bergisch Gladbach, Germany) as described previously. ${ }^{16}$ In brief, peripheral blood mononuclear cells (PBMCs) were isolated from the vaccinees and unvaccinated healthy controls. Non-memory B cells in PBMCs, including $\mathrm{T}$ cells, natural killer cells, monocytes, dendritic cells, granulocytes, platelets and erythroid cells, were depleted using a cocktail of biotinylated antibodies against CD2, CD14, CD16, CD36, CD43 and CD235a (glycophorin A), and anti-biotin microbeads. Subsequently, magnetic labeling of memory B cells with CD27 microbeads were performed for positive selection of memory B cells.

\section{Flow cytometry}

Isolated cells ware analyzed by a standard flow cytometry protocol as described previously. ${ }^{17}$ Briefly, cells were washed with phosphate-buffered saline containing $1 \%$ bovine serum albumin and incubated with surface-staining antibodies. After staining with FITC-conjugated CD27, PE-conjugated CD19 (Miltenyi Biotech) at $4{ }^{\circ} \mathrm{C}$ for $20 \mathrm{~min}$, cells were washed with phosphate-buffered saline and resuspended in $300 \mu$ phosphatebuffered saline. The data were analyzed using FlowJo software (Version 5.7.2; Tree Star, Inc., Ashland, OR, USA).

\section{EBV preparation and immortalization of peripheral blood memory B cells}

EBV for immortalization of human B lymphocytes was prepared from B95-8 cells, as described previously. ${ }^{18}$ In short, B95-8 cells were cultured at a high density of $10^{6}$ cells/ml in RPMI 1640 with $10 \%(\mathrm{v} / \mathrm{v}) \mathrm{FCS}$ at $37^{\circ} \mathrm{C}$ in $5 \% \mathrm{CO}_{2}$. The supernatant was collected and filtered with a sterile filter $(0.45 \mu \mathrm{m}$; Millipore, Billerica, MA, USA) before storing the aliquots in liquid nitrogen. Peripheral blood memory B cells were EBV-transformed according to the protocol, as described previously. ${ }^{19}$ Allogenic mononuclear cells $\left(5 \times 10^{5}\right.$ cells $\left./ \mathrm{ml}\right)$ in RPMI 1640 medium without sera were irradiated by $35 \mathrm{Grey} \mathrm{Co}^{60}$ and plated in 96-well plates (50 000 cells/ $100 \mu \mathrm{l}$ per well) in $30 \%$ FCS. These irradiated cells were used as feeding cells. Using flow cytometry, memory B cells were sorted into a single cell per well into 96-well plates with feeding cells before the addition of $50 \mu \mathrm{l}$ mixture per well containing $48 \mu \mathrm{l}$ of EBV suspension, $1 \mu \mathrm{l}$ of cyclosporine A solution (at the final concentration of $400 \mathrm{ng} / \mathrm{ml}$ ) and $1 \mu \mathrm{l}$ of CpG 2006 (at final concentration of $2.5 \mu \mathrm{g} / \mathrm{ml}$ ). After EBV transformation, immortalized memory B-cell clones were expanded for 28 days with medium changed every 3-5 days. The supernatant of each clone $(100 \mu \mathrm{l})$ was used to screen positive clones specific for 2009 pandemic (H1N1) HA.

\section{Expression of recombinant HA proteins (rHAs)}

rHAs were expressed and purified using the Baculovirus/insect cell system (BD Biosciences, San Diego, CA, USA), as described previously. ${ }^{20,21}$ In brief, the HA ectodomain DNA fragment of A/ Sichuan/1/2009 (H1N1) HA gene was cloned into the transfer vector PacGP67b (BD Biosciences). Linearized baculovirus DNA (BD Biosciences) and the transfer vector PacGP67b were cotransfected into Sf9 cells for the generation of recombinant baculoviruses. Viral supernatant was collected at $72 \mathrm{~h}$ post-infection. rHAs were expressed after three rounds of infection and purified using Ni-NTA chromatography (GE Healthcare, Pittsburgh, PA, USA). Western blotting confirmed the expression and purification of rHAs with anti-His or anti-HA antibodies.

\section{Production of pseudotype influenza virus}

Pseudotype H1N1 viruses were generated as described previously. ${ }^{20,22}$ Briefly, pseudotype influenza viruses were produced 
using a retrovirus-based pseudotype system via the expression of full-length genes of HA and neuraminidase from A/Sichuan/1/ 09, A/Beijing/262/95 and A/Brisbane/59/07, Gag-Pol and a firefly luciferase reporter gene in a $293 \mathrm{~T}$ cell expression system. Viral supernatants were harvested $48 \mathrm{~h}$ post-transfection for the HAI test and viral neutralization assay.

\section{Enzyme-linked immunosorbent assay (ELISA)}

ELISAs were performed to measure the HA-binding activities of sera from vaccinees or MAbs from immortalized memory B cells, as described previously. ${ }^{20}$ Briefly, rHAs were coated on polystyrene plates at $4{ }^{\circ} \mathrm{C}$ overnight followed by blocking with $5 \%$ bovine serum albumin (Sigma, St Louis, MO, USA) at $37{ }^{\circ} \mathrm{C}$ for $1 \mathrm{~h}$. The sera from vaccinees at a dilution of $1: 100$ were incubated in wells at $37^{\circ} \mathrm{C}$ for $1 \mathrm{~h}$. After complete wash, horseradish peroxidase-labeled anti-human $\operatorname{IgG}$ or $\operatorname{IgM}(1: 5000)$ (Sigma) was added at $37{ }^{\circ} \mathrm{C}$ for $1 \mathrm{~h}$. The OPD/ $\mathrm{H}_{2} \mathrm{O}_{2}$ was added to develop color, and the reaction was stopped with $50 \mu \mathrm{l}$ of $\mathrm{H}_{2} \mathrm{SO}_{4}$. Plates were detected at $450 / 630 \mathrm{~nm}$.

\section{Western blot}

rHAs were resolved using SDS-polyacrylamide gel electrophoresis and electrophoretically transferred to a nitrocellulose membrane. After blocking with 5\% skim milk, the blots were probed with anti-His-tag mouse MAbs at room temperature for $1 \mathrm{~h}$. After a complete wash, horseradish peroxidaseconjugated anti-mouse IgG was added and incubated at room temperature for $1 \mathrm{~h}$ before protein detection with the West Pico Chemiluminescent Substrate (Thermo Scientific, Rockford, IL, USA).

\section{HAI assay}

HAI assays of sera or MAbs were performed according to a standard hemagglutination-inhibition protocol..$^{20,23,24}$ Briefly, sera from vaccinees or MAbs from immortalized memory B cells were diluted initially at $1: 5$ and treated overnight with Vibrio cholerae receptor-destroying enzyme (Denka-Seiken, Tokyo, Japan) followed by inactivation for $30 \mathrm{~min}$ at $56{ }^{\circ} \mathrm{C}$ to destroy non-specific inhibitors. Serial dilutions of sera or MAbs were pre-incubated with pseudotype H1N1 virus (4 HA units per well) for $30 \mathrm{~min}$ incubation. To wells, $0.75 \%$ type-O human erythrocytes were added, and the plates were incubated on ice for 30 minutes. Positive seroprotection was set at a geometric mean titer over $1: 40$.

\section{Neutralization assays}

Neutralization assays of sera or MAbs were performed as described previously. ${ }^{21,24}$ Serial dilutions of sera or MAbs were incubated with pseudotype $\mathrm{H} 1 \mathrm{~N} 1$ viruses for $30 \mathrm{~min}$ at room temperature before the mixture was added to MDCK cells in 96-well plates. Infection efficiency was quantified by measuring the luciferase activity in the target cells with an EG\&G Berthold Microplate Luminometer LB96V. The neutralization activity of sera or MAbs was analyzed according to the equation: $(\mathrm{A}-\mathrm{B}) /$ $\mathrm{A} \times 100 \%$. ' $\mathrm{A}$ ' represents the value of positive wells that contained only pseudotype viruses, and 'B' represents the value of wells that contained the mixture of testing serum samples and pseudotype viruses.

\section{Statistical analysis}

Data are presented as the mean \pm s.d. of triplicate experiments. Unpaired Student's $t$-test was used for statistical evaluation of the differences among the diverse human MAbs or human sera in HA-binding assays, HAI or neutralizing assays. $P$ values of less than 0.05 were considered statistically significant.

\section{RESULTS}

\section{Vaccination of volunteers with 2009 H1N1 vaccine PANFLU.1}

To generate high viral neutralizing MAbs against 2009 pandemic H1N1 strains, we applied a novel strategy using EBV to immortalize peripheral blood memory B cells from vaccinees who received the $2009 \mathrm{H} 1 \mathrm{~N} 1$ vaccine PANFLU.1 (Figure 1). Because PANFLU.1 appeared to be more effective against H1N1 virus infection in young adults, ${ }^{25,26}$ we selected 19 healthy volunteers between 24 and 45 years of age for screening vaccinees with high viral neutralizing sera. Fourteen volunteers were vaccinated with one dose of PANFLU.1 containing $15 \mu \mathrm{g}$ of HA antigen, and five healthy unvaccinated volunteers were used as a negative control.

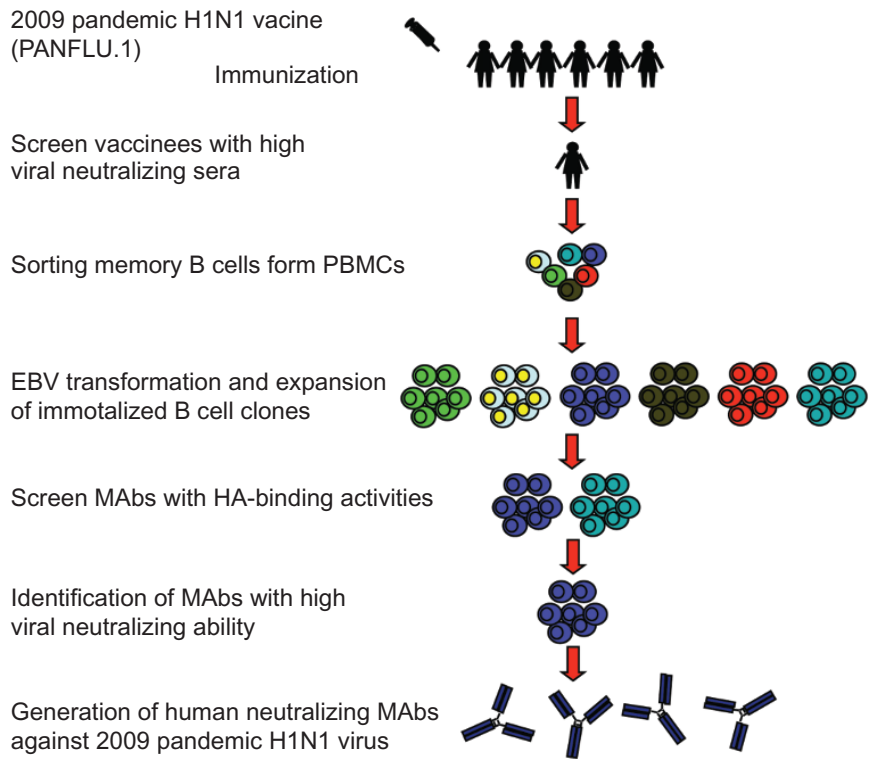

Figure 1 Schematic diagram of strategy for generating high viral neutralizing MAbs from EBV-immortalized peripheral blood memory B cells. Healthy young adults were vaccinated with 2009 pandemic H1N1 vaccine PANFLU.1 for screening with hyper immune sera. Peripheral blood memory $B$ cells from selected vaccinees were sorted and immortalized with EBV. After expansion of immortalized B cell clones, an ELISA was performed to screen HA-binding specific MAbs. Next, we applied viral neutralizing and hemagglutination inhibition assays to identify MAbs with high viral neutralizing capacities and hemagglutination inhibition activities against 2009 pandemic H1N1 viruses. EBV, Epstein-Barr virus; ELISA, enzyme-linked immunosorbent assay; HA, hemagglutination; MAb, monoclonal antibody; PBMC, peripheral blood mononuclear cell. 
Expression of recombinant HA proteins derived from 2009 pandemic H1N1 virus

Previous studies demonstrated that a majority of neutralizing antibodies against pandemic $\mathrm{H} 1 \mathrm{~N} 1$ viruses were able to bind to highly conserved epitopes in the stalk and head regions of HA proteins. ${ }^{4,27}$ Antibodies against the surface glycoprotein HA are of major importance for protection against infection. Thus, HA is the primary component of currently licensed influenza virus vaccines. ${ }^{2}$ In this study, we used rHAs to screen neutralizing antibodies specific for the 2009 pandemic H1N1 virus. The ectodomain of HA from 2009 pandemic H1N1 virus was fused with a His tag at the C-terminus expressed in baculovirus-infected Sf9 insect cells and purified by metal affinity chromatography. Coomassie blue staining confirmed that the size of rHAs was correct and the purity was greater than $80 \%$ (Figure 2a). Western blotting also confirmed that rHAs were successfully expressed and purified (Figure 2b). A HA assay showed that the rHAs possessed HA comparable activities with the $2009 \mathrm{H} 1 \mathrm{~N} 1$ pandemic vaccine (Figure $2 \mathrm{c}$ and d). These results suggest that the rHAs could be used to screen high affinity HA-specific antibodies specific for the 2009 pandemic H1N1 virus.

\section{Selection of vaccinees with hyperimmune sera}

To verify the vaccination efficiency of PANFLU.1, the sera from 14 vaccinees were analyzed using an ELISA to assess HA-binding and using a neutralizing capacity assay at different time points after vaccination (Figure $3 \mathrm{a}$ ). The sera from the vaccinated group had significantly higher HA-binding activity and stronger neutralizing capacity for the 2009 pandemic pseudotype H1N1 virus compared with unvaccinated healthy controls $(P<0.01$, ANOVA) (Figure 3a and $\mathrm{b})$. The HA-binding activities and neutralizing capacities for the 2009 pandemic pseudotype H1N1 virus remained at relatively high levels between 2 and 8 weeks after vaccination (Figure $3 \mathrm{a}$ and $\mathrm{b}$ ). This finding is consistent with previous studies. ${ }^{9,19}$ At 8 weeks postvaccination, sera from all 14 vaccinees had high binding to rHA and neutralizing capacities for the 2009 pandemic pseudotype $\mathrm{H} 1 \mathrm{~N} 1$ virus (Figure $3 \mathrm{c}$ and $\mathrm{d}$ ). In contrast, all five unvaccinated healthy controls had no antibody response to either rHA or the 2009 pandemic pseudotype H1N1 virus. Notably, vaccinee 4 (V4) had approximately twofold higher HA-binding activities compared with other vaccinees (Figure 3c). A HAI assay showed that sera from all 14 vaccinees were HAI-positive $\left(\mathrm{HAI}^{+}\right.$) (defined as positive at a minimum sera dilution $>1: 40$ ), while sera from the five unvaccinated healthy volunteers were HAI-negative $\left(\mathrm{HAI}^{-}\right)$ (Figure 3e). The numbers of memory B cells $\left(\mathrm{CD} 19^{+} \mathrm{CD} 27^{+}\right.$ cells) from PBMCs were also significantly increased in all 14 vaccinees compared with those in the unvaccinated volunteers (Figure 3f). Intriguingly, the number of memory B cells among the PBMCs from V4 was the highest in all vaccinees, correlating with the rHA binding assay result (Figure $3 \mathrm{f}$ and c). These results taken together suggest that vaccination with the 2009 pandemic H1N1 vaccine PANFLU.1 could efficiently induce high affinity HA-specific antibodies with strong neutralizing capacities for the 2009 pandemic pseudotype H1N1 virus.

\section{Immortalization of peripheral blood memory B cells from vaccinees with hyperimmune sera}

To immortalize peripheral blood memory B cells, $\mathrm{CD} 19^{+} \mathrm{CD} 27^{+}$ memory B cells were isolated from PBMCs from V4 and two vaccinees (V1 and V7) at 8 weeks post-vaccination. The purity of $\mathrm{CD} 19^{+} \mathrm{CD} 27^{+}$memory B cells attained more than $90 \%$ after flow cytometry sorting (Figure 4a). $\mathrm{CD} 19^{+} \mathrm{CD} 27^{+}$memory $\mathrm{B}$ cells were individually sorted into 96-well plates (single cell per well) containing EBV, CpG2006 and feeding cells.
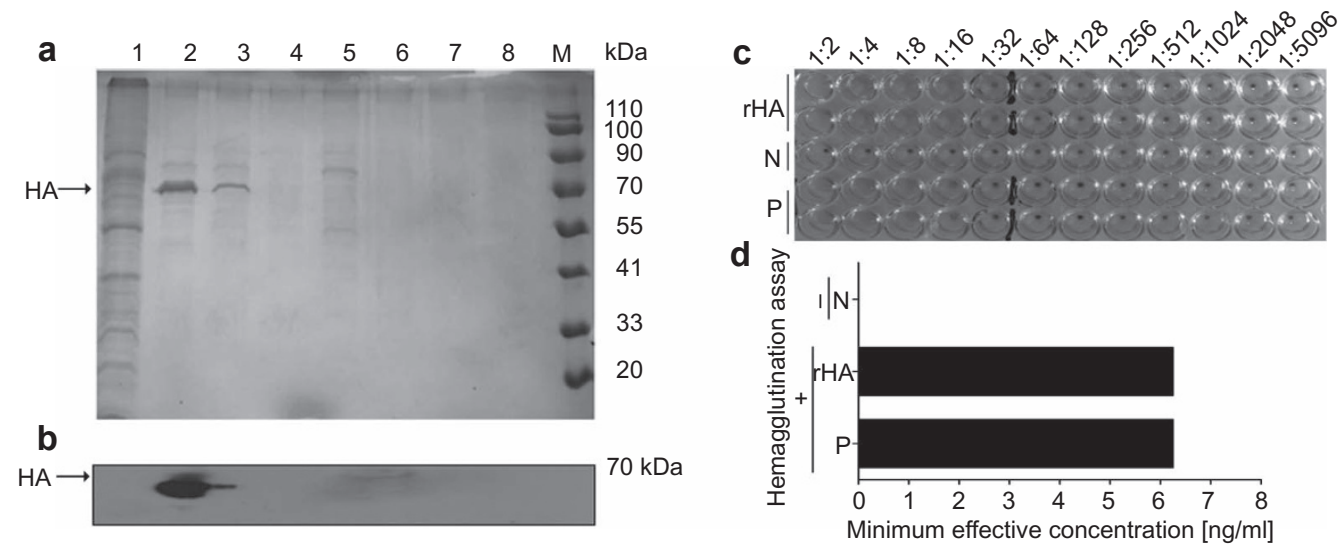

Figure 2 Expression of rHAs. (a) rHA expression and purification was detected by Coomassie blue staining of 10\% polyacrylamide SDS-PAGE. Lane 1, normal Sf9 lysate. Lanes 2 and 3, purified rHAs with 10 and $1 \mu \mathrm{g}$, respectively. Lane 4, non-specific protein control for His-tag affinity chromatography. Lane 5, normal Sf9 culture supernatant. Lanes 6-8, SF-900 medium as negative controls. Lane M, protein marker. (b) Western blot analysis for expressed $\mathrm{rHAs}$ with anti-His antibodies. (c, d) Detection of hemagglutination activity of purified rHA. The 2009 pandemic $\mathrm{H} 1 \mathrm{~N} 1$ vaccine was used as a positive control $(\mathrm{P})$ and $0.1 \% \mathrm{BSA}$ as a negative control $(\mathrm{N})$. BSA, bovine serum albumin; $\mathrm{HA}$, hemagglutination; rHA, recombinant hemagglutination; $\mathrm{Sf9}$, spodoptera frugiperda. 

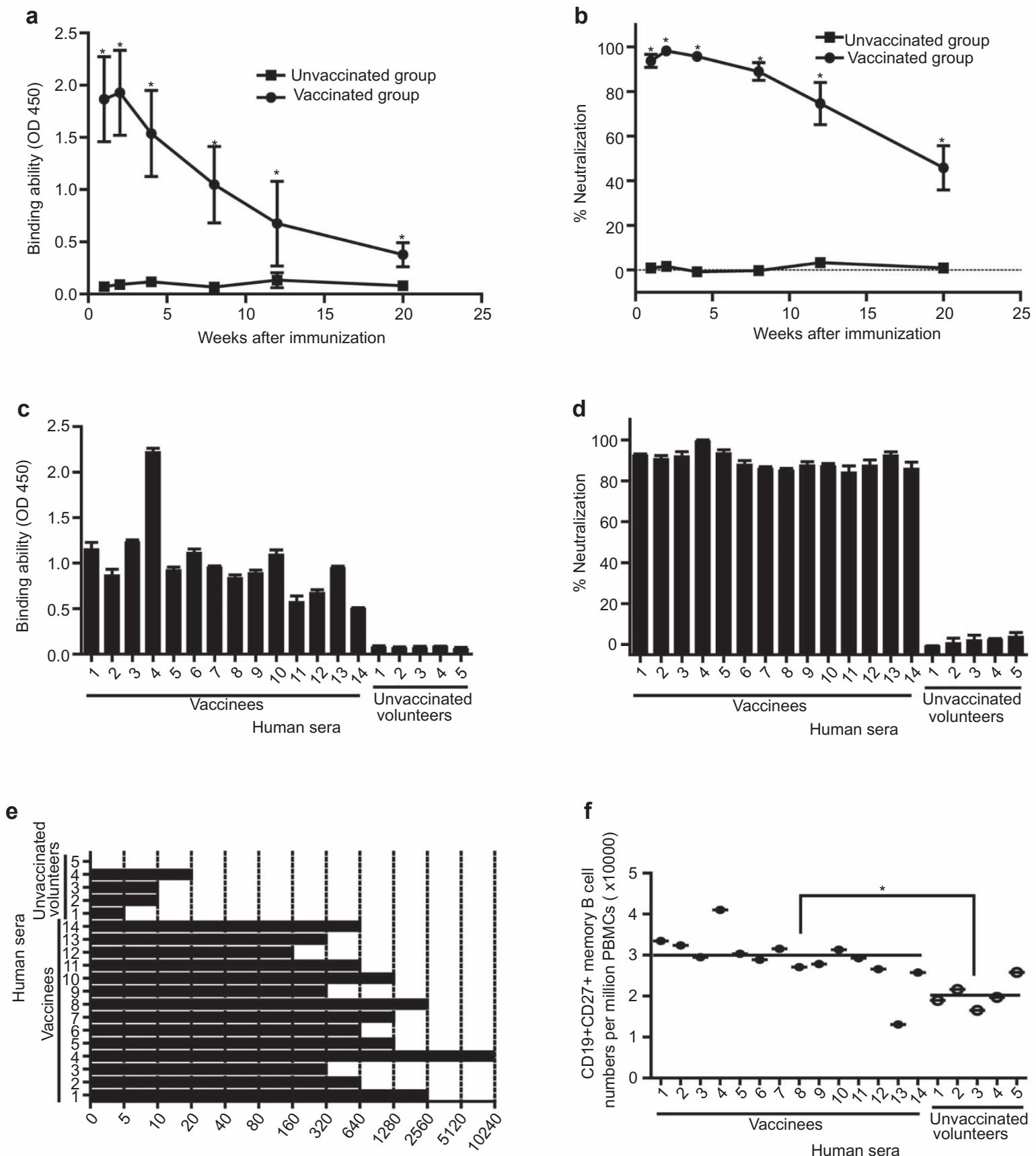

Figure 3 Screening vaccinees with hyperimmune sera. The sera and CD19 ${ }^{+} \mathrm{CD} 27^{+}$memory B cells from the vaccination group (includes 14 vaccinees designated as $\mathrm{v} 1$ to $\mathrm{v} 14$ ) and the unvaccinated group (includes five healthy unvaccinated volunteers designated as N1 to N5) were examined at various time points after immunization. (a) ELISA analysis of the rHA binding activities of sera from vaccinees at different postvaccination time points ( 1 week (w), $2 \mathrm{w}, 4 \mathrm{w}, 8 \mathrm{w}, 12 \mathrm{w}$ and $20 \mathrm{w}$ ). $* P<0.01$, compared with controls. (b) ELISA analysis of neutralizing activities of sera from vaccinees at post-vaccination time points. The values at each time point represent the mean \pm s.d. $* P<0.01$, compared with controls. (c) rHA binding activities of sera from vaccinees at $8 \mathrm{w}$ post-vaccination. (d) The neutralizing activities of sera from vaccinees at $8 \mathrm{w}$ post-vaccination. (e) $\mathrm{HAI}$ of sera from vaccinees at the time point of day 21 post-vaccination. Each serum sample was tested in duplicate or triplicate in ELISA analysis. (f) Flow cytometry analysis of the numbers of memory B cells (CD19+CD27 ${ }^{+}$cells) per million PBMCs from vaccinees. ${ }^{*} P<0.01$, compared with unvaccinated control. ELISA, enzyme-linked immunosorbent assay; HAI, hemagglutination inhibition; PBMC, peripheral blood mononuclear cell; rHA, recombinant hemagglutination.

EBV-transformed memory B cells were amplified to become immortalized B cell clones in 2-4 weeks (Figure $4 \mathrm{~b}$ ). The mean frequency of immortalization of memory B cells from three vaccinees was $56.67 \%$. However, V7 showed relatively higher immortalization frequency of memory B cells compared with V1 and V4 (Figure 4c). 
a

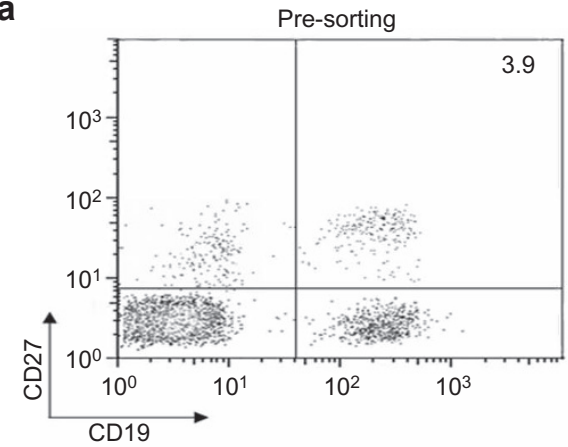

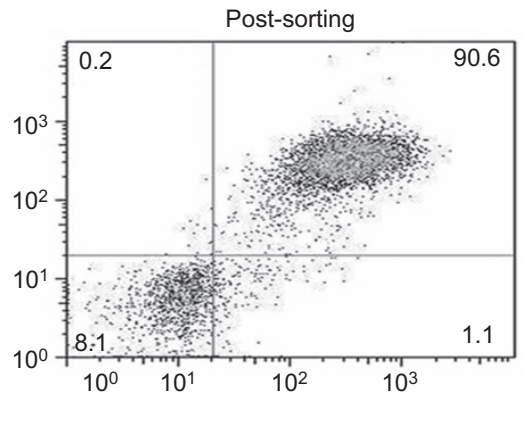

b
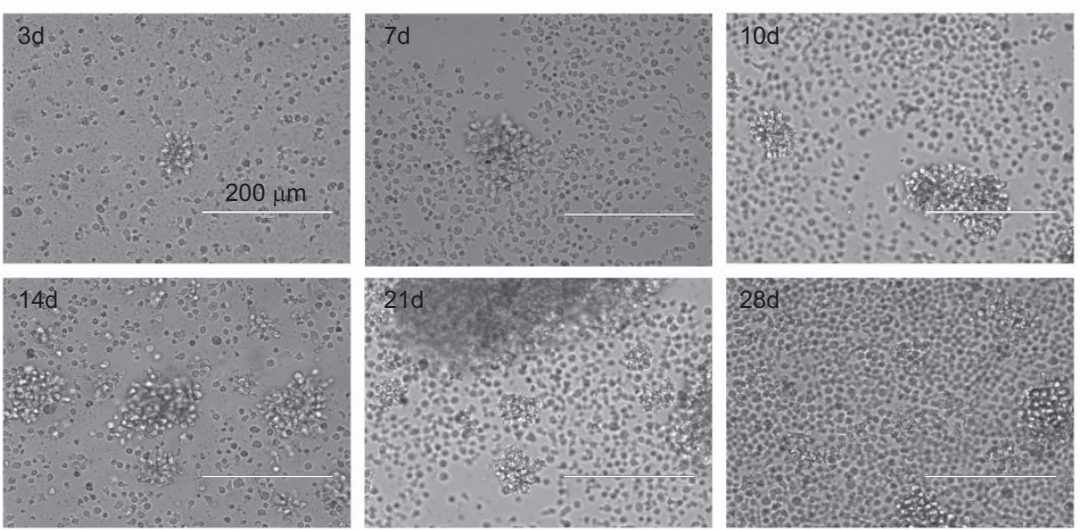

C

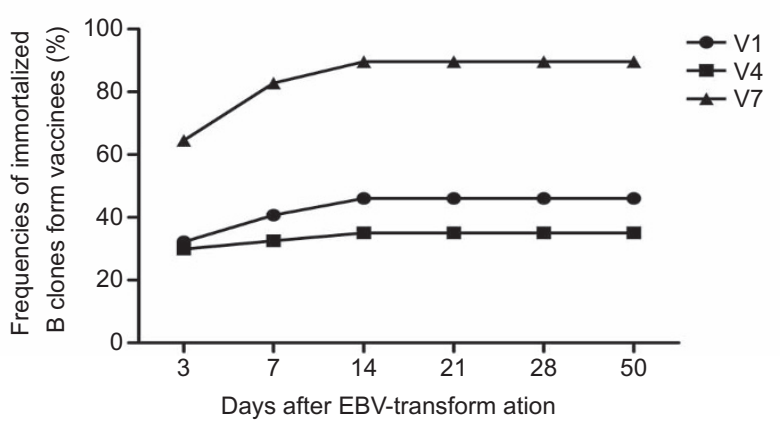

Figure 4 Immortalization of memory $\left(\mathrm{CD} 19^{+} \mathrm{CD} 27^{+}\right) \mathrm{B}$ cells by EBV-transduction. Flow cytometry analysis of memory $\mathrm{B}$ cells from three selected vaccinees before and after sorting. (a) Flow cytometry plots showed that the purity of $C D 19^{+} \mathrm{CD} 27^{+} \mathrm{B}$ cells increased from $3.9 \%$ to $90.6 \%$ after sorting. (b) Representative images of EBV-immortalized B cells at different time points after EBV transduction. (c) The frequencies of immortalized memory B-cell clones from V1, V4 and V7 were calculated using the formula: immortalized B-cell clones/all B cell clones (per vaccinee). EBV, Epstein-Barr virus.

Screening immortalized memory B-cell clones secreting HAspecific MAbs

To identify HA-specific antibody-producing immortalized memory B-cell clones, 13090 immortalized memory B-cell clones were screened by using ELISA to measure the HAbinding activities. Of these, 617 clones were identified as positive clones (Figure $5 \mathrm{a}-\mathrm{c}$ ), 281 of which were derived from V4 (Figure 5a). The mean frequency of HA-specific antibody-producing memory B-cell clones was $4.82 \%$ (Figure 5d). An HAI assay was performed to test the hemagglutination inhibition activities of 617 clones, and 32 clones, including 16 clones derived from V4, were $\mathrm{HAI}^{+}$(Figure 5e).

\section{Identification of high viral neutralizing MAbs}

A neutralizing assay was performed to identify immortalized memory B-cell clones secreting high viral neutralizing MAbs against 2009 pandemic H1N1 virus. Nine MAbs were identified with neutralizing capacities against the pseudotype H1N1 viruses $(P<0.01)$ compared with controls (Figure 6a), 
a

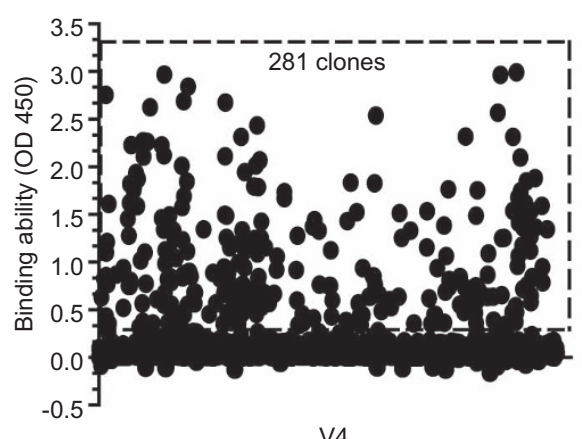

b

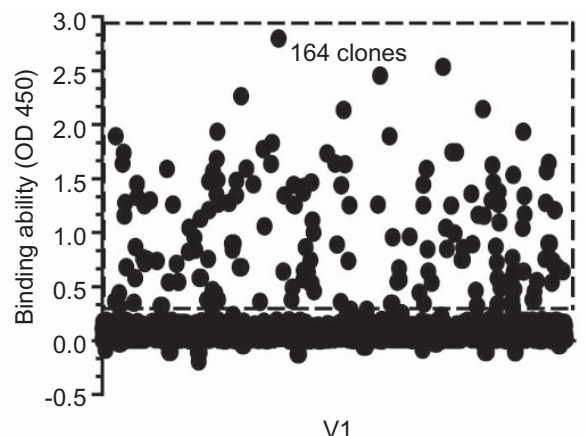

C

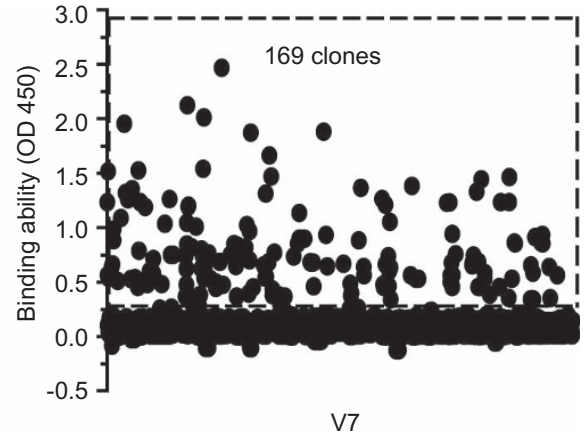

d

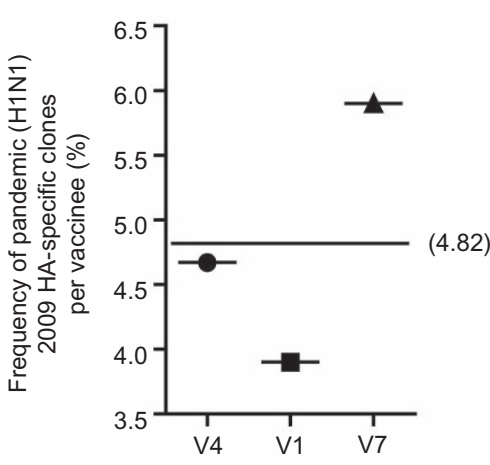

e

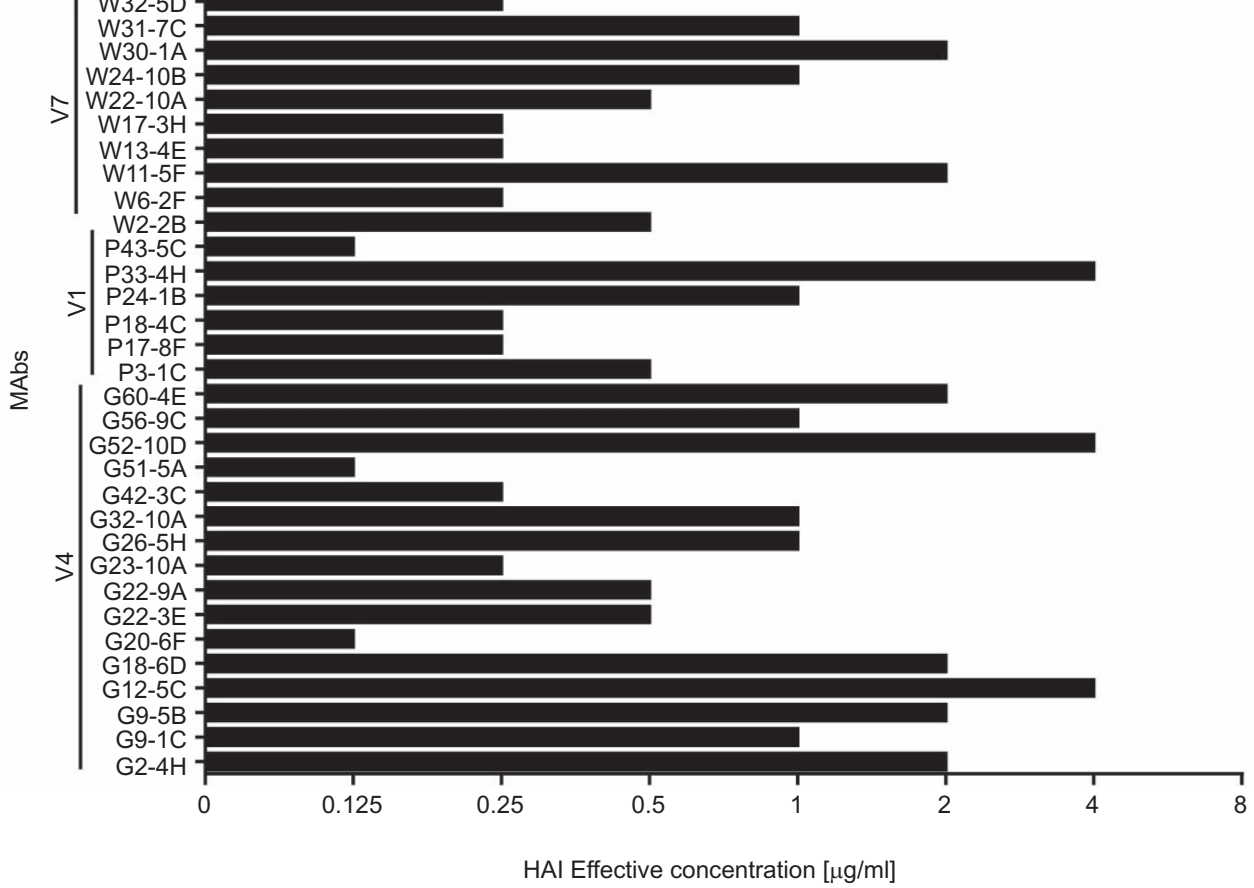

Figure 5 Screening immortalized memory B-cell clones producing high affinity HA-specific MAbs. ELISA analysis of rHA binding activities of MAbs secreted by immortalized memory B-cell clones from V1 (b), V4 (a) and V7 (c). The Optical Density (OD) values $\geqslant 2$.1-fold over negative control value were considered positive clones. If the OD values of negative controls were less 0.05 , they were calculated as 0.05 . (d) Analysis of the frequency of rHA-specific immortalized memory B-cell clones. (e) $\mathrm{HAl}^{+}$B cell clones identified from vaccinees V1, V4 and V7. ELISA, enzyme-linked immunosorbent assay; HA, hemagglutination; HAI, hemagglutination inhibition; MAb, monoclonal antibody; rHA, recombinant hemagglutination.

representing $0.061 \%$ frequency of total memory B cells (Figure 6b). These 9 MAbs showed high affinity to rHA (Figure 6c) $(P<0.01)$ compared with controls. More importantly, nine MAbs also possessed high viral neutralizing capacities against two different strains of pseudotype H1N1 viruses (A/Beijing/262/95 and A/Brisbane/59/07 strains) (Figure 6d and 6e) $(P<0.01)$ compared with controls, suggesting a broad viral neutralizing capacity to 2009 pandemic H1N1 influenza viruses. Furthermore, seven MAbs were $\mathrm{HAI}^{+}$and two MAbs were $\mathrm{HAI}^{-}$(Figure 6f). Finally, seven MAbs were finally identified with both high viral neutralizing capacities and HAI activities against 2009 pandemic H1N1 influenza viruses.

\section{DISCUSSION}

In this study, we demonstrated a rapid and efficient strategy for the generation of human MAbs by immortalizing peripheral blood memory B cells from young adults vaccinated with the 2009 pandemic H1N1 influenza vaccine. Nine immortalized memory B-cell clones were identified as secreting high viral neutralizing antibodies toward the pandemic $\mathrm{H} 1 \mathrm{~N} 1$ virus. Seven MAbs displayed both strong viral neutralizing capacities and HAI activities.

Compared to other techniques for generating human MAbs, such as humanizing murine MAbs or selection of antibody fragments from phage-display libraries, ${ }^{10-13}$ our strategy has two major advantages. First, the seven immortalized memory 
a
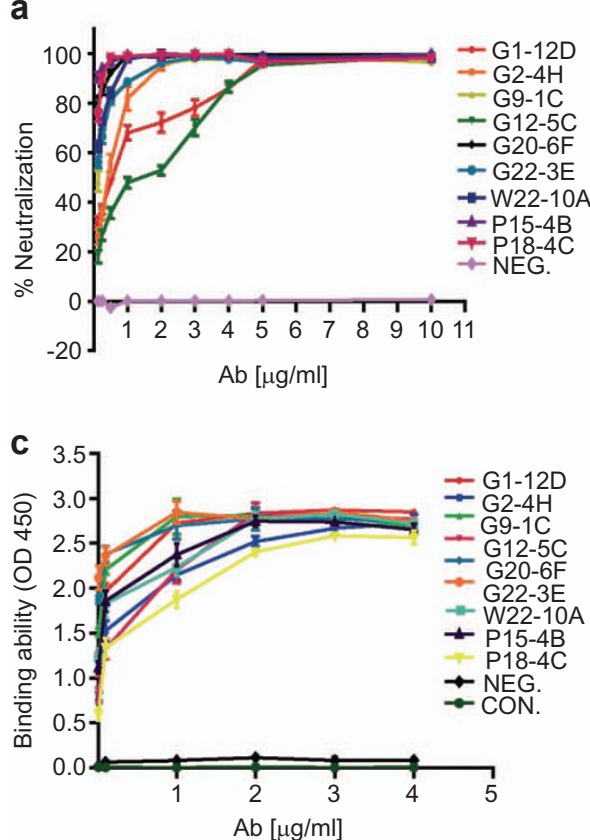

e

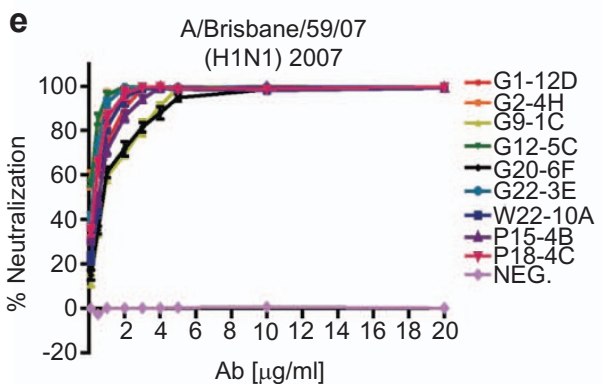

b

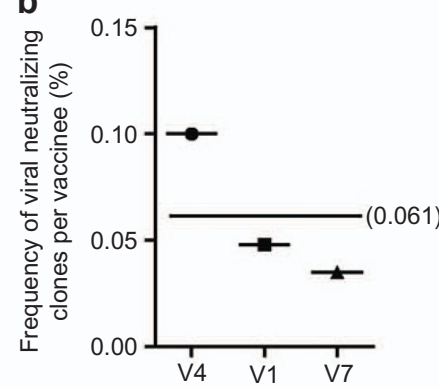

d
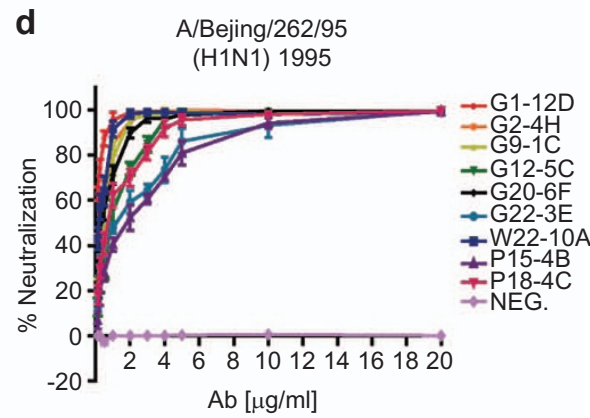

f

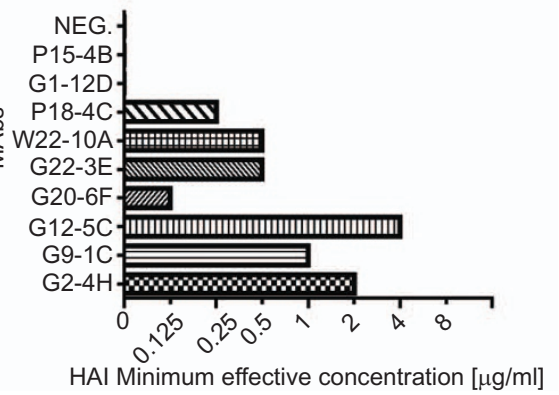

Figure 6 Identification of high viral neutralizing MAbs against 2009 pandemic H1N1 viruses. (a) Neutralization capacity analysis of nine MAbs with pseudotype H1N1 viruses at different MAbs concentrations (10, 5, 4, 3, 2, 1, 0.5, 0.25 and $0.125 \mu \mathrm{g} / \mathrm{ml})$. (b) The frequencies of neutralizing MAbs from vaccinees V1, V4 and V5. (c) HA-binding activities of nine MAbs at different MAb concentrations $(0.01,0.1,1,2,3 \mathrm{and} 4 \mu \mathrm{g} / \mathrm{ml})$. (d, e) Neutralization analysis of nine MAbs with two strains of pseudotype H1N1 viruses (A/Beijing/262/95 and A/Brisbane/59/07) at different concentrations $\left(20,10,5,4,3,2,1,0.5,0.25\right.$ and $0.125 \mu \mathrm{g} / \mathrm{ml}$ ). (f) $\mathrm{HAl}$ analysis of nine neutralizing MAbs showed seven $\mathrm{HAl}^{+} \mathrm{MAbs}$ and two HAl ${ }^{-} \mathrm{MAbs}$. $\mathrm{HA}$, hemagglutination; $\mathrm{HAI}$, hemagglutination inhibition; MAb, monoclonal antibody.

B-cell clones were 2009 pandemic H1N1 influenza strainspecific memory B cells proliferating in response to the specific vaccine, and they were not resting memory $B$ cells, which are composed of a range of $\mathrm{B}$-cell clones generated by a variety of previous exposures. Second, compared to generating immortalized B-cell clones from influenza-infected patients, it appears to be more efficient to obtain high neutralizing activity MAbs against pandemic influenza H1N1 viruses from selected healthy vaccinees. Serum from V4 had significantly higher titers of neutralizing activity and HA-binding activity than those from any other vaccinees. In fact, six of nine immortalized memory B-cell clones were derived from V4. These results indicate that the antibody response to pandemic H1N1 2009 vaccine may be variable among vaccinated individuals, though we could not exclude the possibility of pre-existence of anti-HA antibodies in V4 due to previous H1N1 viral infection. Therefore, it was necessary to screen the vaccinees with hyperimmune sera before the generation of human MAbs from immortalized memory B-cell clones.

Recently, several studies reported success in generating neutralizing antibodies from peripheral blood plasma cells of vaccinated individuals or infected patients., ${ }^{4,28,29}$ However, because peripheral blood plasma cells could only survive 1-2 weeks after vaccination or infection, they identified the genes encoding human variable regions using single-cell RT-PCR of plasmablasts and subsequently expressed the antibodies in Escherichia coli. ${ }^{15}$ Clearly, the MAbs from immortalized human memory B-cell clones are more natural and stable in viral neutralizing activities over a lifetime and are more suitable for clinical applications.

In this study, we found that, although nine identified MAbs displayed high viral neutralizing activities, two of them were negative in the HAI assay, indicating that the epitopes recognized by these two MAbs may be located at the stem region of 
HA proteins, consistent with previous descriptions. ${ }^{4,15,28,30-35}$ Escape mutation, overlapping the HA peptide-binding assay and competition ELISA may be useful for identifying their epitopes in future studies. We also found that $4.82 \%$ of MAbs isolated from immortalized memory B cells had HAbinding activity, but only $0.24 \%$ had HAI activity and only $0.061 \%$ of MAbs could efficiently neutralize H1N1 viruses. These data indicate that the frequency of memory B cells specifically reactive against 2009 pandemic $\mathrm{H} 1 \mathrm{~N} 1$ viruses is low but detectable in the whole memory B-cell repertoire, consistent with the studies reported previously., ${ }^{9,15}$

Passive serotherapy with polyclonal or MAbs has been widely used for infectious diseases such as respiratory syncytial virus, Hepatitis A and B, anthrax, smallpox, meningitis and the plague. ${ }^{10,36-39}$ In the case of influenza, serotherapy with MAbs has also provided prophylactic or therapeutic protection in mice and other animal models. ${ }^{4,40,41}$ In this study, we developed seven MAbs with high neutralizing capacities against 2009 pandemic H1N1 viruses. Our findings suggest that these MAbs may provide potent therapeutics for treating severe influenza cases and protecting high-risk populations such as children, the elderly and immunodeficient individuals.

In addition, compared with conventional methods, the strategy used in this study is a rapid method for generating human neutralizing MAbs from peripheral blood memory B cells with high efficiency and accuracy. This method bypasses the need for generating humanized hybridomas and can deliver large numbers of antibodies, making it easier to select the most favorable antibodies with high affinity or titer and epitope specificity. Moreover, this strategy would also be applicable for rapid generation of neutralizing antibodies in a pandemic outbreak of other emerging pathogens and contributes to the serotherapy field for infectious or autoimmune diseases.

\section{ACKNOWLEDGEMENTS}

This work was funded by National Natural Science Foundation of China (31070785) and US Civilian Research \& Development Foundation (CHB1-31056-BE-11).

1 de Whalley P, Pollard A. Pandemic influenza A (H1N1) 2009 vaccination in children: a UK perspective. J Paediatr Child Health 2013; 49: E183-E188.

2 Garten RJ, Davis CT, Russell CA, Shu B, Lindstrom S, Balish A et al. Antigenic and genetic characteristics of swine-origin 2009 $A(H 1 N 1)$ influenza viruses circulating in humans. Science 2009; 325: 197-201.

3 Brockwell-Staats C, Webster R, Webby R. Diversity of influenza viruses in swine and the emergence of a novel human pandemic influenza A (H1N1). Influenza Other Respir Viruses 2009; 3: 207-213.

4 Wrammert J, Koutsonanos D, Li G, Edupuganti S, Sui J, Morrissey M et al. Broadly cross-reactive antibodies dominate the human B cell response against 2009 pandemic H1N1 influenza virus infection. J Exp Med 2011; 208: 181-193.

5 Liang $\mathrm{X}$, Wang $\mathrm{H}$, Wang J, Fang $\mathrm{H}, \mathrm{Wu} \mathrm{J}$, Zhu $\mathrm{F}$ et al. Safety and immunogenicity of 2009 pandemic influenza A H1N1 vaccines in China: a multicentre, double-blind, randomised, placebo-controlled trial. Lancet 2010; 375: 56-66.
6 Kozbor D, Roder J, Chang T, Steplewski Z, Koprowski H. Human antitetanus toxoid monoclonal antibodies secreted by EBV-transformed human B cells fused with murine myelorna. Hybrodoma 1982; 1: 323-328.

7 Karpas A, Dremucheva A, Czepulkowski B. A human myeloma cell line suitable for the generation of human monoclonal antibodies. Proc Natl Acad Sci USA 2001; 98: 1799-1804.

8 Steinitz M, Klein G, Koskimies S, Makel O. EB virus-induced B lymphocyte cell lines producing specific antibody. Nature 1977; 269: 420-422.

9 Jin A, Ozawa T, Tajiri K, Obata T, Kondo S, Kinoshita K et al. A rapid and efficient single-cell manipulation method for screening antigenspecific antibody-secreting cells from human peripheral blood. Nat Med 2009; 15: 1088-1092.

10 Brekke $\mathrm{OH}$, Sandlie I. Therapeutic antibodies for human diseases at the dawn of the twenty-first century. Nat Rev Drug Discov 2003; 2: 52-62.

11 Jones P, Dear P, Foote J, Neuberger M, Winter G. Replacing the complementarity-determing regions in a human antibody with those from a mouse. Nature 1986; 321: 522-525.

12 McCafferty J, Griffiths A, Winter G, Chiswell D. Phage antibodies: filamentous phage displaying antibody variable domains. Nature 1990; 348: 552-554.

13 Sui J, Hwang W, Perez S, Wei G, Aird D, Chen L et al. Structural and functional bases for broad-spectrum neutralization of avian and human influenza A viruses. Nat Struct Mol Biol 2009; 16: 265-273.

14 Green L. Antibody engineering via genetic engineering of the mouse: XenoMouse strains are a vehicle for the facile generation of therapeutic human monoclonal antibodies. J Immunol Methods 1999; 231: 11-23.

15 Li G, Chiu C, Wrammert J, McCausland M, Andrews S, Zheng N et al. Pandemic H1N1 influenza vaccine induces a recall response in humans that favors broadly cross-reactive memory B cells. Proc Natl Acad Sci USA 2012; 109: 9047-9052.

16 Henn A, Rebhahn J, Brown M, Murphy A, Coca M, Hyrien 0 et al. Modulation of single-cell IgG secretion frequency and rates in human memory B cells by CpG DNA, CD4OL, IL-21, and cell division. J Immunol 2009; 183: 3177-3187.

$17 \mathrm{Xi} \mathrm{X}$, Guo Y, Chen H, Xu C, Zhang H, Hu H et al. Antigen specificity of gammadelta $T$ cells depends primarily on the flanking sequences of CDR3delta. J Biol Chem 2009; 284: 27449-27455.

18 James E, Shaw R , Leung K. Growth of B95-8 cells and expression of Epstein-Barr Virus lytic phase in serum-free medium. J Virol 1987. 61: 4033-4037.

19 Traggiai E, Becker S, Subbarao K, Kolesnikova L, Uematsu Y, Gismondo $\mathrm{M}$ et al. An efficient method to make human monoclonal antibodies from memory B cells: potent neutralization of SARS coronavirus. Nat Med 2004; 10: 871-875.

$20 \mathrm{Li} \mathrm{Z,} \mathrm{Ma} \mathrm{C,} \mathrm{Liu} \mathrm{Z,} \mathrm{He} \mathrm{W.} \mathrm{Serologic} \mathrm{cross-reactivity} \mathrm{among} \mathrm{humans} \mathrm{and}$ birds infected with highly pathogenic avian influenza A subtype H5N1 viruses in China. Immunol Lett 2011; 135: 59-63.

21 Li Z, Liu Z, Ma C, Zhang L, Su Y, Gao G et al. Identification of amino acids in highly pathogenic avian influenza $\mathrm{H} 5 \mathrm{~N} 1$ virus hemagglutinin that determine avian influenza species specificity. Arch Virol 2011; 156: 1803-1812.

22 Lin X, Zhou J, Zhang Y, Wu J, Zhang F, Li Z et al. Oseltamivir boosts 2009 H1N1 virus infectivity in vitro. Biochem Biophys Res Commun 2009; 390: 1305-1308.

23 Hoffmann E, Lipatov A, Webby R, Govorkova E, Webster R. Role of specific hemagglutinin amino acids in the immunogenicity and protection of H5N1 influenza virus vaccines. Proc Natl Acad Sci USA 2005; 102: 12915-12920.

24 Yu X, Tsibane T, McGraw P, House F, Keefer C, Hicar M et al. Neutralizing antibodies derived from the B cells of 1918 influenza pandemic survivors. Nature 2008; 455: 532-536.

25 Wu J, Xu F, Lu L, Lu M, Miao L, Gao T et al. Safety and effectiveness of a 2009 H1N1 vaccine in Beijing. N Eng/ J Med 2010; 363: 2416-2423.

26 Qiu Y,Yin W. Safety and immunogenicity of Sinovac's prototype pandemic influenza H5N1 vaccines: a review on clinical trials. Influenza Other Respi Viruses 2008; 2: 237-242. 
27 Terajima M, Cruz J, Co MD, Lee JH, Kaur K, Wilson P et al. Complement-dependent lysis of influenza a virus-infected cells by broadly cross-reactive human monoclonal antibodies. J Virol 2011; 85: 13463-13467.

28 Corti D, Voss J, Gamblin SJ, Codoni G, Macagno A, Jarrossay D et al. A neutralizing antibody selected from plasma cells that binds to Group 1 and Group 2 influenza A Hemagglutinins. Science 2011; 333: 850-856.

29 Hancock K, Veguilla V, Lu X, Zhong W, Butler EN, Sun H et al. Crossreactive antibody responses to the 2009 pandemic $\mathrm{H} 1 \mathrm{~N} 1$ influenza virus. N Engl J Med 2009; 361: 1945-1952.

30 Corti D, Suguitan AJ, Pinna D, Silacci C, Fernandez-Rodriguez B, Vanzetta $\mathrm{F}$ et al. Heterosubtypic neutralizing antibodies are produced by individuals immunized with a seasonal influenza vaccine. J Clin Invest 2010; 120: 1663-1673.

31 Ekiert DC, Bhabha G, Elsliger MA, Friesen RH, Jongeneelen M, Throsby $\mathrm{M}$ et al. Antibody recognition of a highly conserved influenza virus epitope. Science 2009; 324: 246-251.

32 Ekiert DC, Friesen RH, Bhabha G, Kwaks T, Jongeneelen M, Yu W et al. A highly conserved neutralizing epitope on group 2 influenza A viruses. Science 2011; 333: 843-850.

33 Ekiert $\mathrm{D}$, Wilson I. Broadly neutralizing antibodies against influenza virus and prospects for universal therapies. Curr Opin Virol 2012; 2: 134-141.

34 Krause JC, Tumpey TM, Huffman CJ, McGraw PA, Pearce MB, Tsibane $T$ et al. Naturally occurring human monoclonal antibodies neutralize both 1918 and 2009 pandemic influenza A (H1N1) viruses. J Virol 2010; 84: 127-130.

35 Throsby M, van den Brink E, Jongeneelen M, Poon LL, Alard P, Cornelissen $\mathrm{L}$ et al. Heterosubtypic neutralizing monoclonal antibodies cross-protective against $\mathrm{H} 5 \mathrm{~N} 1$ and $\mathrm{H} 1 \mathrm{~N} 1$ recovered from human $\operatorname{lgM}^{+}$memory B cells. PLOS ONE 2008; 3: e3942.

36 An Z. Monoclonal antibodies - a proven and rapidly expanding therapeutic modality for human diseases. Protein Cell 2010; 1: 319-330.

37 Brocks B, Kraft S, Zahn S, Noll S, Pan C, Schauer M et al. Generation and optimization of human antagonistic antibodies against TIMP-1 as potential therapeutic agents in fibrotic diseases. Hum Antibodies 2006; 15: 115-124.

38 Zuidscherwoude M, van Spriel AB. The origin of IgE memory and plasma cells. Cell Mol Immunol 2012; 9: 373-374.

39 Zheng J, Liu Y, Lau Y, Tu W. Gammadelta-T cells: an unpolished sword in human anti-infection immunity. Cell Mol Immunol 2013; 10: 50-57.

40 Renegar KB, Small PA Jr, Boykins LG, Wright PF. Role of IgA versus IgG in the control of influenza viral infection in the murine respiratory tract. J Immunol 2004; 173: 1978-1986.

41 Wang X, Chan CC, Yang M, Deng J, Poon VK, Leung VH et al. A critical role of $\mathrm{IL}-17$ in modulating the $\mathrm{B}$-cell response during $\mathrm{H} 5 \mathrm{~N} 1$ influenza virus infection. Cell Mol Immunol 2011; 8: 462-468. 